\title{
Introducing Potential Radiative Forcing (PRF) for quantifying the Earth radiation budget
}

\author{
Kazuaki Kawamoto a and Tadahiro Hayasaka ${ }^{\mathrm{b}}$ \\ ${ }^{a}$ Faculty of Environmental Studies, Nagasaki University, 1-14 Bunkyo-machi, Nagasaki, Japan \\ ${ }^{b}$ Center for Atmosphere and Oceanic Studies, Tohoku University, 6-3 Aoba, Aramaki, Aoba, Sendai, Japan
}

\begin{abstract}
In order to investigate the contributions of atmospheric factors to variability in surface shortwave irradiance $(S)$ under both cloud-free and cloudy sky conditions, we defined the sensitivity of $S$ to differential increases from given values of cloud, aerosol, and water vapor as potential radiative forcing (PRF). The expected change in $S$ due to one factor would be the product of the PRF and the change in that factor. We formulated the PRF from a simple equation and described the geographical features of the PRF over China in January and July. We noted some shortcomings, but confirmed the usefulness of the PRF for determining the relative contributions. In particular, we suggested that accurate knowledge of aerosol absorption properties was critical for quantitative radiation budget estimates.
\end{abstract}

Keywords: Radiation budget, radiative forcing, clouds, aerosols, water vapor

PACS: 92.60.Mt, 92.60.nf

\section{INTRODUCTION}

The surface shortwave irradiance $(S)$ drives climate processes through dynamical, thermodynamic, and radiative processes. Scattering and absorption by air molecules, aerosols and cloud particles influence $S$, before it reaches the surface. Because the effects of these factors vary temporally and spatially, $S$ shows large variability over different timescales. Previous studies have reported that $S$ decreased for several decades before the 1990s and then began to increase (the so-called global dimming and brightening), although the observation points were limited ${ }^{1}$. The reason for this long-term variation is still unclear and is the subject of active discussion. If it is assumed that other atmospheric parameters remained constant, the reports of decreased $S$ between 1960 and 1990 appear contradictory to the decreasing total amount of cloud found in ground measurements over most regions of China ${ }^{2}$. Large quantitative differences still exist between the observed values of $S$ and those produced by simulations of radiative forcing that include anthropogenic aerosols. A new index that represents the sensitivity of $S$ to changes in factors such as clouds, aerosols, and water vapor would be useful because it would allow variability in $S$ to be estimated from observed changes in these factors. Here, we defined an index of potential radiative forcing (PRF) based on the sensitivity of $S$ to differential change in various factors, described the geographical features of PRF over the region including China, and discuss the contributions of the factors to variability in $S$.

\section{DEFINING POTENTIAL RADIATIVE FORCING (PRF)}

Values of $S$ (here, $0.2-5 \mu \mathrm{m}$ ) are influenced by various factors, including clouds, aerosols, water vapor, and surface albedo, depending on the solar incidence at the point of interest. The relationships between $S$ and atmospheric factors can be expressed by simple equations. For a non-reflecting surface, $S$ is formulated as

$$
S=S_{0}\left[\left(1-A_{c}\right) T_{a}+A_{c} T_{c}\right],
$$

where $S_{0}$ is the incident solar irradiance at the top of the atmosphere, which varies with the season, latitude, state of the Earth's orbit, and solar activity; and $T_{a}$ and $T_{c}$ are transmittance under cloud-free and cloudy sky conditions, respectively. The cloud-free sky is defined as a condition with aerosols and water vapor, and no clouds. The total amount of cloud $A_{c}$ is defined as the fraction of sky occupied by cloudiness.

The differential of Eq. (1) is 
$\Delta S=S_{0}\left[\left(1-A_{c}\right) \Delta T_{a}+A_{c} \Delta T_{c}+\left(T_{c}-T_{a}\right) \Delta A_{c}\right]$.

According to Eq. (2), cloud-free and cloudy factors do not alter $S$ independently. If $A_{c}$ is small, the change in $T_{a}$ strongly affects $S$, but the change in $T_{c}$ does not have a large effect on $S$. We are interested in the potential radiative forcing (PRF), which we define as the sensitivity of $S$ to the differential change in each factor (cloud optical depth $\tau_{\mathrm{c}}$, aerosol optical depth $\tau_{\mathrm{a}}$, amount of water vapor $w$, and $A_{c}$ ), according to the following respective expressions:

$$
\begin{aligned}
& \frac{\partial S}{\partial \tau_{c}}=S_{0} A_{c} \frac{\partial T_{c}}{\partial \tau_{c}}, \\
& \frac{\partial S}{\partial \tau_{a}}=S_{0}\left[\left(1-A_{c}\right) \frac{\partial T_{a}}{\partial \tau_{a}}+A_{c} \frac{\partial T_{c}}{\partial \tau_{a}}\right], \\
& \frac{\partial S}{\partial w}=S_{0}\left[\left(1-A_{c}\right) \frac{\partial T_{a}}{\partial w}+A_{c} \frac{\partial T_{c}}{\partial w}\right], \\
& \frac{\partial S}{\partial A_{c}}=S_{0}\left(T_{c}-T_{a}\right)
\end{aligned}
$$

The expected change in $S$ due to one factor is obtained as the product of PRF and the change in that factor, and the total change in $S$ is estimated by the summation. The PRF is defined for unit optical depth for $\tau_{\mathrm{c}}$ and $\tau_{\mathrm{a}}, 1 \%$ for $A_{c}$, and $1 \mathrm{~mm}$ for $w$. As is evident from the above equations, the PRF depends on a combination of the values of the factors and $S_{0}$ according to $A_{c}$. The PRF is always negative, and becomes stronger (more influential on $S$ ), when the factor values are small and $S_{0}$ is large. Hereafter, 'strong' and 'weak' imply 'large (more negative)' and 'small (less negative)' absolute values for the PRF. Feedback processes among these factors (e.g., indirect aerosol effects) are not explicitly treated in the above equations because of their complexity and the poor understanding of the processes involved. The effects of ozone and other minor constituents on $S$ are not considered because they are negligible. The radiation quantities were computed using a general radiative transfer code RSTAR- $5 b^{3}$.

\section{GEOGRAPHICAL FEATURES OF PRF}

\section{a) Data sets}

Because atmospheric factors generally vary greatly with the season, we used monthly instead of annual mean data for the analyses. The monthly averaged PRF in January and July over the region containing China and neighboring countries $\left(75^{\circ}-135^{\circ} \mathrm{E}, 20^{\circ}-55^{\circ} \mathrm{N}\right)$ was calculated using the RSTAR-5b radiation code. The inputs were $\tau_{\mathrm{c}}$ at visible wavelength, $A_{c}$ from the International Satellite Cloud Climatology Project (ISCCP) data sets ${ }^{4}, \tau_{\mathrm{a}}$ at $0.55 \mu \mathrm{m}$ from the Moderate Resolution Imaging Spectroradiometer (MODIS) products ${ }^{5}$, and $w$ archived by the European Center for Medium-range Weather Forecasts (ECMWF). Four-year (2002-2005) climatologies were constructed with $2.5^{\circ}$ resolution. This period was selected based on the availability of the advanced MODIS instrument. We used the ISCCP-derived ground albedo in our calculations, although Eqs. (1) to (6) assumed a non-reflecting surface for simplicity.

\section{b) Clouds}

Figure 1 shows the PRF due to $\tau_{c}$. Hereafter, the upper and lower panels are for January and July, respectively, in all figures. In the upper panel, the PRF was weak over the north $\left(>45^{\circ} \mathrm{N}\right)$ and the south $\left(<30^{\circ} \mathrm{N}\right)$. We attributed the former effect to the moderately thick clouds and small $S$ there, and the latter to the large $\tau_{c}$ in the east and small $A_{c}$ in the west, respectively. There was a stronger PRF in the central area, caused by optically thin clouds. In the lower panel, these clouds were generally weak over the south and the Korean Peninsula, where moderately optically thick clouds were present. The inland desert and mountainous regions had a particularly strong PRF because of the optically thin clouds there. This was also the case over the East China Sea.

Figure 2 illustrates the PRF due to $A_{c}$. As shown in the upper panel, the PRF over the north was relatively weak in comparison with that over the southeast and the East China Sea. The lower panel shows a strong PRF over the Tibetan Plateau, which was caused by moderately large $\tau_{\mathrm{c}}$ and small $\tau_{\mathrm{a}}$. The PRF in the central arid region was weak because of the small $\tau_{\mathrm{c}}$ and moderate $\tau_{\mathrm{a}}$ there. These effects were associated with the contrast between $T_{c}$ and $T_{a}$, as expressed in Eq. (6). 


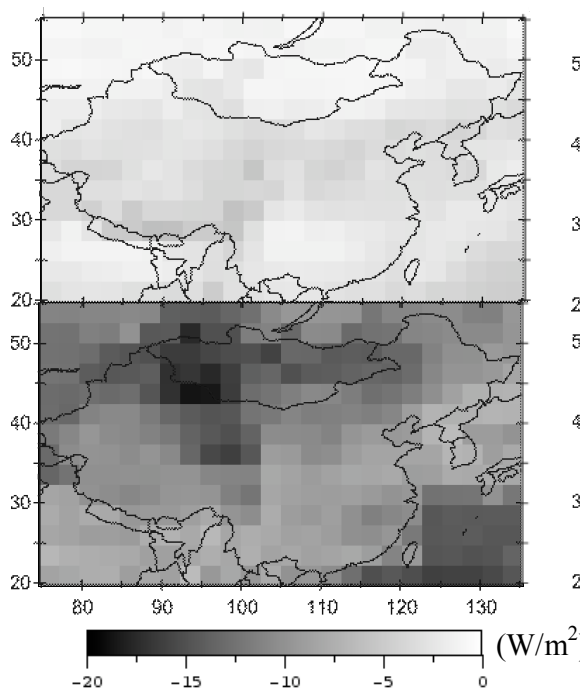

Figure 1

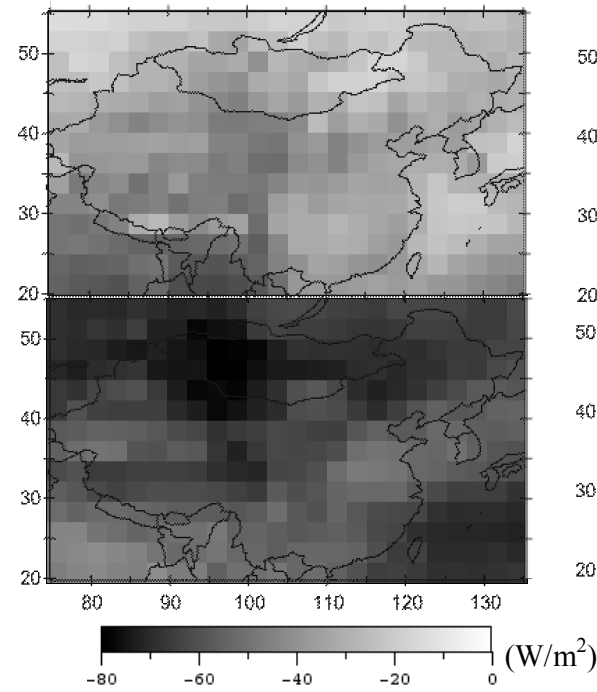

Figure 3

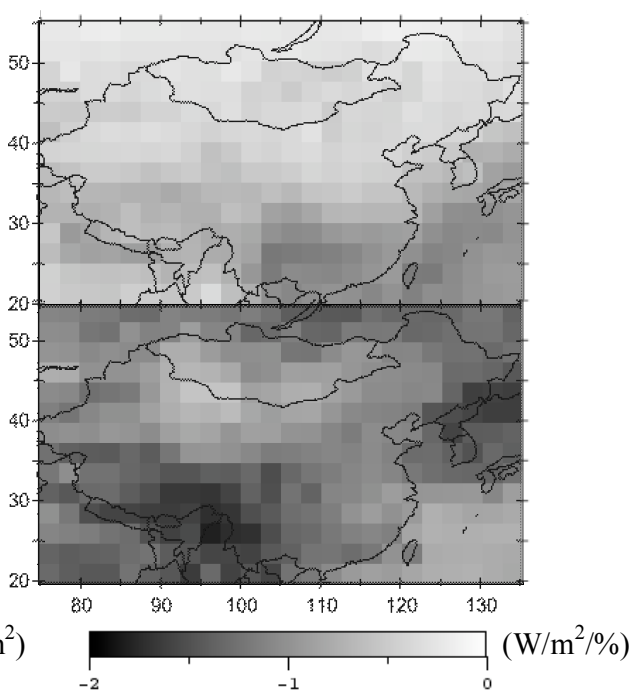

Figure 2

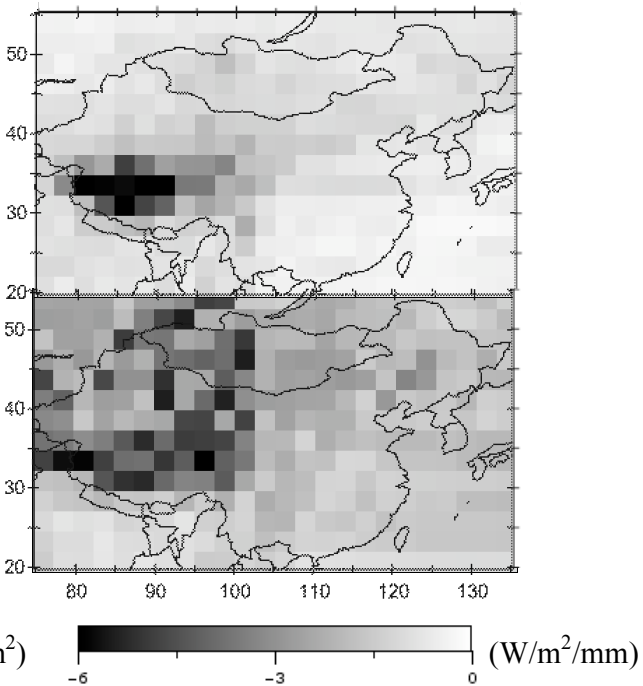

Figure 4

c) Aerosols

Figure 3 shows the PRF due to $\tau_{a}$. In the upper panel, the PRF was weaker over a narrow latitude band in the north; the PRF in the western part of the southern region was stronger, whereas that in the other regions appeared moderate. In contrast to that in the January case, the PRF over the north and the East China Sea was stronger, and that over the south was weaker in the lower panel. The PRF over the middle of the northern area was particularly strong because of the thin clouds there. The aerosol absorption property is largely decided by the imaginary part $\left(\mathrm{k}_{\mathrm{i}}\right)$ of the refractive index. We set $\mathrm{k}_{\mathrm{i}}$ to 0.02 , corresponding to moderately absorptive substances such as dust or carbonaceous aerosols.

\section{d) Water vapor}

Figure 4 shows the PRF due to $w$. In January, the PRF over Tibet was strong, reflecting dry conditions. Over most other regions, however, the PRF was rather weak. In July, the PRF from the north to the west, where $w$ was small, was relatively strong. The effects in other regions were generally weak. Although values of $w$ had similar 
geographical distributions, different total amounts for the two months. Because of these results and the seasonal change in $S_{o}$, the seasonal difference in PRF due to $w$ was much smaller than that due to other factors.

\section{CONCLUDING REMAKS}

We defined a PRF index based on the sensitivity of $S$ to differential increases in affecting factors (clouds, aerosols, and water vapor). We then described the geographical distributions of the factors and PRF in January and July. Our results demonstrate the usefulness of the PRF index in all sky conditions (including cloudy sky) as well as cloud-free-sky cases addressed by some field campaigns ${ }^{6}$. Additionally, Our method has potential for use in modeling studies, although further refinement is needed for comprehensive elucidation of the variability in $S$.

There are several shortcomings to our method. First, a fixed value for $\mathrm{k}_{\mathrm{i}}$ was assumed over the entire region, even though $\mathrm{k}_{\mathrm{i}}$ can vary widely, especially over China, depending on the aerosol composition ${ }^{7}$. The parameters related to aerosol absorptivity, including size distributions, need to be properly determined. Second, feedback processes among clouds, aerosols, and water vapor were not considered. For example, the indirect aerosol effect is one of the most uncertain phenomena in climate problems ${ }^{8}$. It is likely that other unidentified feedbacks also exist. Improving the knowledge of these feedbacks is critical.

\section{ACKNOWLEDGMENTS}

One of the authors (K. K.) was also supported by a Japan Society for the Promotion of Science (JSPS) grant-inaid for young scientists (B) and scientific research (C) and the President's discretionary funds of Nagasaki University. We used atmospheric and surface products provided by the ISCCP and MODIS science teams.

\section{REFERENCES}

1. M. Wild, et al., Science, 308, 847-850 (2005).

2. Y. Qian, D.P. Kaiser, L.R. Leung, and M. Xu, Geophys. Res. Lett., 33, L01812, doi:10.1029/2005GL024586. (2006)

3. T. Nakajima and M. Tanaka, J. Quant. Spectrosc. Radiant. Transfer, 40, 51-69 (1988).

4. W. B. Rossow and E. N. Duenas, Bull. Am. Meteorol. Soc., 85, 167-172 (2004).

5. Y. J. Kaufman, D. Tanré, and O. Boucher, Nature, 419, 215-223 (2002).

6. T. Nakajima et al., J. Geophys. Res., 108(D23), 8658, doi:10.1029/2002JD003261 (2003).

7. X. Yu, Cheng, T., Chen, J. and Y. Liu, Atmos. Environ., 40, 5787-5797 (2006).

8. K. Kawamoto, T. Hayasaka, I. Uno and M. Ohara, J. Geophys. Res., 111, D19201, doi:10.1029/2005JD006919. (2006). 